\title{
An Atypical Inguinal Hernia in a 9-Month-Old Girl - Case Report and Ultrasound Findings
}

\section{Introduction}

The incidence of pediatric inguinal hernias has been cited in other studies to be between $0.8 \%$ and $4.4 \%$, and the male to female ratio in a clinical series containing 6361 pediatric ingiunal hernias in infants and children under the age of 18 was $5: 1$. In female infants, inguinal hernias contained an ovary in $15 \%$ of cases. The presence of a irreducible ovary increases the risk of hernial strangulation, with strangulation rate estimates of $2 \%$ to $33 \%$ (S. Ein, et.al., Journal of Pediatric Surgery, 41.5; 2006; 980-86.), presenting a risk of necrosis of the ovary. Differential diagnoses in female infants are numerous and include hydrocele of the canal of Nuck, femoral hernia, epidermal inclusion cysts, cystic lymphangiomas, lymphadenopathy, lymphadenitis, rhabdomyosarcoma, and metastatic tumor (K. Hennelly et.al., The Journal of Emergency Medicine, 40.1; 2011; 33-36). This suggests the importance of rapid and precise diagnosis, with ultrasound (US) possibly being a helpful noninvasive preoperative diagnostic tool for non-reducible inguinal masses.

In this case report, we present a female infant with an inguinal hernia containing a torqued and strangulated ovary diagnosed by US.

\section{Case}

A 9-month-old female infant was admitted to her local hospital with a painful lump in the right groin. The girl had a short previous history of fever and emesis but was still relatively well with no acute symptoms. Initial examinations showed a smooth, movable, 3 by $1 \mathrm{~cm}$ tender mass in the right groin with no miscoloration or redness. Blood tests were normal. Over the course of the following day, concerns grew, as her general appearance worsened and lymph node or inguinal hernia was considered as a differential diagnosis. US was performed bedside by the pediatrician, who raised the suspicion of irreducible inguinal hernia and the patient was transferred to our hospital. The patient, now clearly in pain and very fussy, was eval- uated by the pediatric surgeon and a new US examination was requested. US was performed by the radiologist on call with a GE Logiq E9 system and a $9 \mathrm{MHz}$ linear transducer with small parts preset. This revealed a well circumscribed heterogenous mass with cystic areas ( $\triangleright$ Fig. $\mathbf{1}$ ) and sparse flow by color Doppler ( $\triangleright$ Fig. 2 ). The lump measured 3 by $1.5 \mathrm{~cm}$ and communicated with the inguinal canal by a stalk with clearly visible flow. Pain was intensified by touching the lump, which was irreducible and without peristalsis corresponding to herniation of an ovary.

Surgery was performed using open inguinal incision, sparing the ovary as manual reduction was impossible. As suspected, the hernia sac contained an ischemic ovary, which was twisted four times around the axis of its blood supply. After detorsion, the ovary was repositioned, and the hernia sac was closed. The girl quickly recovered and was soon discharged.

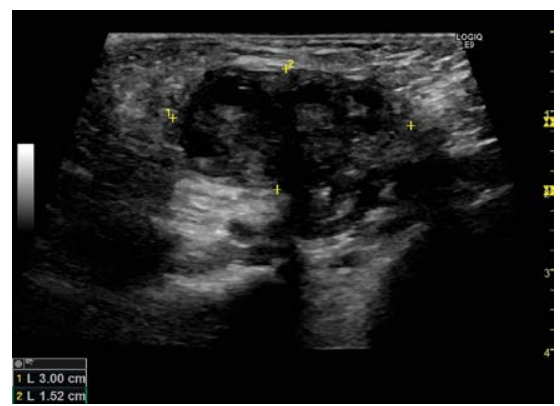

- Fig. 1 A well-circumscribed heterogeneous mass with cystic areas communicating with the inguinal canal - an ovarian hernia.

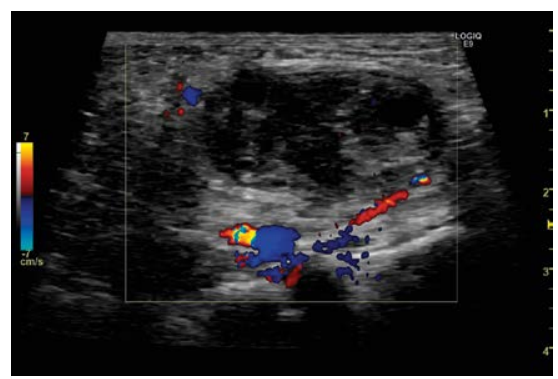

- Fig. 2 Visible flow in the ovarian vessels in the inguinal canal.

\section{Discussion}

We have described the case of a 9-monthold girl with a herniated, torquated and strangulated ovary in the right inguinal canal. Almost all inguinal hernias in female infants are indirect and are thought to arise from failure in the closure of the processus vaginalis. The resulting canal is termed the canal of Nuck and may allow for an inguinal hernia to develop. A herniated ovary in itself can appear quite harmless, but complications can arise when the ovary twists around the axis of its blood supply, thereby constricting blood flow and strangulating the ovary. Strangulation poses the risk of necrosis, infection, peritonitis and possibly infertility.

Even though an ovary is not an uncommon finding in female pediatric hernias, differential diagnosis may be difficult. Additionally, as the range of clinical presentations of a herniated ovary in infants can vary, further diagnostic measures are often needed. US is a safe, noninvasive diagnostic tool for inguinal masses. Visualizing a mass with hypoechoic follicular areas and without any peristalsis should prompt the clinician to consider the possible diagnosis of a herniated ovary. Color Doppler enables visualization of blood flow within the ovary, thereby enabling evaluation of the severity of strangulation (R Auslender et.al., Ultrasound in Obstetrics \& Gynecology, 34.2; 2009; 208-11). However, it should be noted that not all strangulated ovaries initially display an absence of arterial blood flow. Therefore, the evaluation of the patient should always be based on integration of the US examination and the clinical findings (J. Robertson et.al., The Journal of Emergency Medicine, 52.4; 2017; 449-56).

Surgical detorsion and repositioning or complete removal of the affected ovary are the most common procedures. In non-hernia-related cases of a strangulated ovary in children, detorsion and repositioning of the ovary is preffered in order to maximize preservation of viable tissue (C. Spinelli et. al, Current Opinion in Obstetrics and Gynecology, 27.5; 2015; 320-25). Most likely, the same recommendation can be applied to the strangulated ovary within a hernia, 
suggesting preservative surgery as the method of choice.

In conclusion, as this case illustrates, a herniated ovary should be considered as a possible diagnosis in the pediatric patient with an inguinal mass. US can be useful in identifying the mass and, along with the clinical presentation, in raising suspicion of possible strangulation.

Authors

Jonathan Cohen ${ }^{1}$, Susanne Reinhardt ${ }^{2}$, Dorte Levin Pedersen ${ }^{3}$, Caroline Ewertsen 4

\section{Affiliations}

1 Kobenhavns Universitet Panum Instituttet, Panum Instituttet, Kobenhavn, Denmark

2 Rigshospitalet, Department of Pediatric Surgery, Copenhagen, Denmark

3 Zealand University Hospital, Department of Radiology, Køge, Denmark

4 Copenhagen University Hospital, Rigshospitalet, Department of Radiology, Copenhagen OE, Denmark

\section{Correspondence}

Mr. Jonathan Cohen

Kobenhavns Universitet Panum Instituttet

Panum Instituttet

Blegdamsvej 3B

2200 Kobenhavn

Denmark

Tel : +4561260782

mailjonathancohen@gmail.com
Bibliography

DOI https://doi.org/10.1055/a-0732-4669

Ultrasound Int Open 2018; 4: E117-E118

(c) Georg Thieme Verlag KG Stuttgart · New York ISSN 2199-7152

\section{(c) (i) (ㅇ) $\ominus$}

